\title{
Radiative heat transfer from a black body to dielectric nanoparticles
}

\author{
Yann Chalopin, ${ }^{1}$ Hichem Dammak, ${ }^{2, *}$ Marine Laroche, ${ }^{3}$ Marc Hayoun, ${ }^{4}$ and Jean-Jacques Greffet ${ }^{3}$ \\ ${ }^{1}$ Laboratoire d'Energétique Moléculaire et Macroscopique, CNRS UPR 288, Ecole Centrale Paris, F-92295 Châtenay-Malabry, France \\ ${ }^{2}$ Laboratoire Structures, Propriétés et Modélisation des Solides, CNRS UMR 8580, Ecole Centrale Paris, F-92295 Châtenay-Malabry, France \\ ${ }^{3}$ Laboratoire Charles Fabry, Institut d'Optique, Univ Paris Sud, CNRS, 2 av A Fresnel, F-91127 Palaiseau, France \\ ${ }^{4}$ Laboratoire des Solides Irradiés, Ecole Polytechnique, CEA-DSM, CNRS, F-91128 Palaiseau, France
}

(Received 12 October 2011; published 13 December 2011)

\begin{abstract}
Heating of dielectric nanoparticles by black-body radiation is investigated by using molecular-dynamics simulation. The thermal interaction with the radiation is modeled by coupling the ions with a random electric field and including a radiation reaction force. This approach shows that the heat is absorbed by the polariton mode. Its subsequent redistribution among other vibration modes strongly depends on the particle size and on temperature. We observe energy trapping in a finite subset of vibrational modes and study the relaxation pathway of $(\mathrm{MgO})_{4}$ by performing a selective excitation with a deterministic force.
\end{abstract}

DOI: 10.1103/PhysRevB.84.224301

PACS number(s): 63.22.Kn, 65.80.-g, 05.10.-a

\section{INTRODUCTION}

Nanoscale energy transport and thermal properties of nanomaterials have been attracting a growing interest toward ever smaller scales since the well-established concepts of macroscopic heat transfer are no longer valid. ${ }^{1,2}$ The vibrational dynamics and the subsequent relaxation of thermal energy between molecules differ significantly in materials for which dimensions are comparable to the characteristic length scales associated to the heat carriers. ${ }^{3,4}$ In these regimes, various effects like coherence, ballistic transport, nonlocality, and dispersion forces, ${ }^{5}$ as well as tunneling of evanescent waves influence the conductive and radiative energy transfers. ${ }^{6-8}$ This opens a wide range of potential applications, such as thermoelectric materials development, ${ }^{9}$ thermophotovoltaics at the nanoscale, ${ }^{10}$ and microelectronics thermal management. ${ }^{1}$ Hence heat relaxation in nanoscale systems is a very active research topic, where thermal properties of phonon systems are predicted with various computational approaches. ${ }^{5,11-14}$ Molecular-dynamics (MD) simulations have proven to be particularly relevant for thermal physics at short scales. ${ }^{15,16}$ Moreover, the anharmonicity of atomic interactions is implicitly taken into account. Nevertheless, MD is based on classical mechanics and therefore its use is limited to a large temperature regime, where classical statistics is valid. The lowtemperature physics, below the classical Debye temperature, is lacking. ${ }^{17}$

We propose here to extend the classical MD by introducing a physical model of the black-body (BB) radiation. We generate a random electric field with a colored noise in order to physically mimic the heating of the vibrational modes of polar dielectric materials in contact with a BB radiation. This BB radiation plays the role of a thermostat and will be referred to hereafter as Planck thermostat (BB-MD).

The structure of the paper is as follows. The microscopic mechanisms of dielectric heating by a BB cavity and the numerical implementation of a Planck radiation model are presented in Sec. II. Section III reports the results of the heating of dielectric $(\mathrm{MgO})_{n}$ cubic rocksalt nanoparticles (NP) up to $1.3 \mathrm{~nm}$ at low temperatures. In Sec. IV, we analyze the vibrational relaxation pathways in $(\mathrm{MgO})_{4}$ by performing a selective excitation with a colored deterministic electric force. This section also provides a detailed discussion on the vibrational relaxation mechanisms of the thermal energy absorbed and the subsequent effect of heat trapping by polar modes.

\section{MODELING THE INTERACTION OF A NANOPARTICLE WITH THE BLACK-BODY RADIATION}

\section{A. Model}

The BB radiation-the transfer of thermal energy by electromagnetic waves-is often introduced with the Planck distribution. This distribution provides the frequency dependence of the intensity radiated by a cavity in a thermodynamic equilibrium state. When integrated, the energy emitted from the BB cavity is given by

$$
\int_{-\infty}^{\infty} I(|\omega|, T) \frac{d \omega}{2 \pi}
$$

where

$$
I(\omega, T)=\frac{\omega^{2}}{\pi c^{3}}\left(\frac{\hbar \omega}{e^{\hbar \omega / k_{B} T}-1}\right) .
$$

According to the Wiener-Khintchin theorem, the timecorrelation function of the electric field can be related to the power spectral density as

$$
\left\langle E_{\alpha}(t) E_{\beta}(t+\tau)\right\rangle=\delta_{\alpha \beta} \int_{-\infty}^{+\infty} I_{E}(|\omega|, T) e^{-i \omega \tau} \frac{d \omega}{2 \pi},
$$

and the frequency dependence of the electric component can be obtained using the power spectral density,

$$
I_{E}(\omega, T)=\frac{1}{3 \epsilon_{0}} I(\omega, T) .
$$

$\left\langle E_{\alpha}\right\rangle=0$ for each Cartesian component $\alpha(\alpha=x, y$, or $z) . \delta_{\alpha \beta}$ is the Kronecker symbol indicating that Cartesian coordinates of $\mathbf{E}$ are uncorrelated.

The random electric field is computed following a numerical technique ${ }^{18}$ employed to generate Gaussian random surface with roughness defined by a given correlation function (see the Appendix). In our case, the correlation function is a Planck distribution at a fixed temperature. 


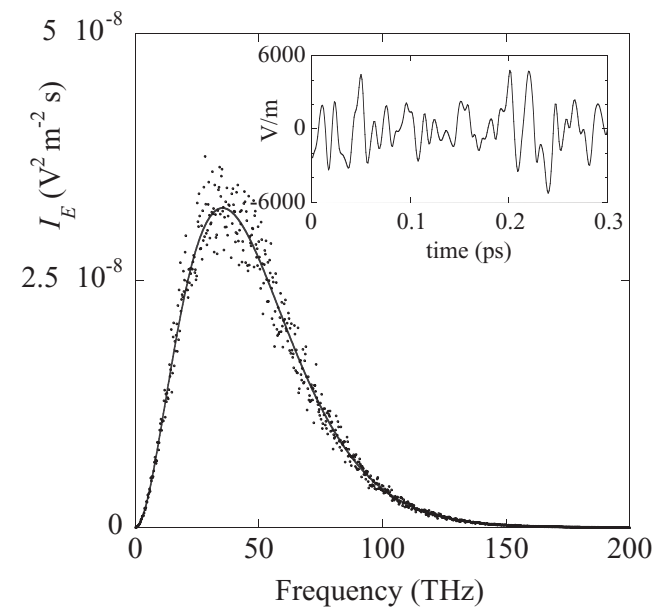

FIG. 1. Spectral density recovered from Fourier transform of the electric field correlation at $T=1000 \mathrm{~K}$. Comparison with the analytical expression given by Eq. (4), dark line. Inset shows a sample of the corresponding time-dependant electric field.

As illustrated on Fig. 1, the Fourier transform of the random field autocorrelation function $\langle E(t) E(t+\tau)\rangle$ obtained in this way follows the spectral density $I_{E}$ prescribed by Eq. (4). It is important to mention that the spatial dependence of $\mathbf{E}$ can be neglected as long as the nanoparticle size remains much smaller than the dominant radiation wavelengths, $\lambda_{\max }$. It corresponds to the wavelength at which the power spectral density spectrum is maximum. $\lambda_{\max }$ remains however much higher than $1 \mu \mathrm{m}$ for temperatures lower than $5000 \mathrm{~K}$; hence neglecting the spatial dependence of $\mathbf{E}$ is justified here.

To simulate the dynamics of a charged nucleus in a molecule, we use the interatomic forces $\mathbf{f}_{i}$, supplemented by the force $q_{i} \mathbf{E}(t)$, where $q_{i}$ is the electric charge. To account for radiative damping, we also introduce a radiation reaction force. This latter force can be written as a function of the third time derivative of the electric dipole moment ${ }^{19}$

$$
\dddot{\mathbf{p}}=\sum_{i}^{N} q_{i} \dddot{\mathbf{r}}_{i},
$$

where $\mathbf{r}_{i}$ is the position of atoms $i$.

The equation of motion of the nucleus $i$ of mass $m_{i}$ interacting with the electric field becomes

$$
m_{i} \ddot{\mathbf{r}}_{i}=\mathbf{f}_{i}+q_{i} \mathbf{E}+m_{i} \eta_{i} \dddot{\mathbf{p}} .
$$

The coefficient $\eta_{i}$ is retrieved from the radiation reaction force calculated in the case of a single moving charge: ${ }^{19}$

$$
\eta_{i}=\frac{q_{i}}{6 \pi \varepsilon_{0} m_{i} c^{3}} .
$$

Finally, the power absorption rate of a nanoparticle with a polarizability spectra $\alpha(\omega)$ in thermal interaction with the Planck radiation is given by

$$
\langle\dot{Q}\rangle=\int_{-\infty}^{\infty}|\omega| I(|\omega|, T) \operatorname{Im}\{\alpha(|\omega|)\} \frac{d \omega}{2 \pi},
$$

so that the heating of the system at an equilibrium temperature is ensured by the electric random field acting as a thermostat.

\section{B. Validation}

To check the validity of the model, we consider a single one-dimensional harmonic dipole defined by a particle of mass $m$ with an angular frequency $\omega_{0}$ obeying

$$
m \ddot{x}=-m \omega_{0}^{2} x+q E+m \eta q \dddot{x} .
$$

For this simple case, the average potential energy at thermal equilibrium is given by

$$
\frac{1}{2} m \omega_{0}^{2}\left\langle x^{2}\right\rangle=\frac{1}{2} u\left(\omega_{0}^{\prime}, T\right),
$$

where

$$
u(\omega, T)=\frac{\hbar \omega}{e^{\hbar \omega / k_{B} T}-1}
$$

and

$$
\omega_{0}^{\prime}=\omega_{0}\left[1-\frac{5}{8}\left(q \eta \omega_{0}\right)^{2}\right],
$$

using the approximation $q \eta \omega_{0} \ll 1$.

Equations (10) and (12) show that the equilibrium energy equals that of a quantum harmonic oscillator $\left(\omega_{0}^{\prime}\right)$. Note that the relative frequency shift $\left(\omega_{0}^{\prime}-\omega_{0}\right) / \omega_{0}$ is typically around $10^{-24}$ for such a diatomic molecule so that it can be neglected.

Another interesting feature is the characteristic time $\tau$ required to reach thermal equilibrium. It is of about $1 /\left(q \eta \omega_{0}^{2}\right) \sim$ $10^{-2} \mathrm{~s}$. In practice, equilibrium needs to be reached within times shorter than $10^{-9} \mathrm{~s}$. A possible strategy to speed up the convergence rate driven by $\tau$ is to balance the charge $q$ by a constant factor $Z$ to enhance the coupling strength with the cavity. This effective charge reduces the transient regime by a factor $Z^{2}$ without perturbating the dynamics of the steady state. The modified set of forces acting on each charge becomes

$$
q E \Rightarrow Z q E
$$

and the corresponding radiation damping term is

$$
m \eta q \dddot{x} \Rightarrow \frac{Z^{2} q^{2}}{6 \pi \varepsilon_{0} c^{3}} \dddot{x} .
$$

$Z$ is however restricted by the condition that guarantees a negligible eigenfrequency shift [Eq. (12)], which can be typically realized by taking $Z \ll 10^{8}$. Figure 2 reports the perfect agreement between the equilibrium average energy obtained numerically for the harmonic dipole and that calculated with the Bose-Einstein distribution $u\left(\omega_{0}, T\right)$.

\section{HEATING DYNAMICS OF (MGO) ${ }_{n}$ NANOPARTICLES}

From the interaction model presented in Sec. II, we aim at capturing the physical mechanisms from which thermally IRactivated vibrational modes couple with radiation and transfer their energy to other vibrational eigenmodes. We will call this process vibrational relaxation in the following discussions.

In this work, we consider $(\mathrm{MgO})_{n}$ rocksalt cubic nanoparticles and choose the interatomic potentials proposed by Matsui. ${ }^{20}$ The symmetry group of MgO NPs is $T_{d}$. Table I reports the fraction of polar modes for $n=4,32$, and 108 . They account for $40 \%-50 \%$ of the total modes.

We calculated the polarizability spectrum, $\alpha(\omega)$, and the vibrational density of states $g(\omega)$ from standard MD 


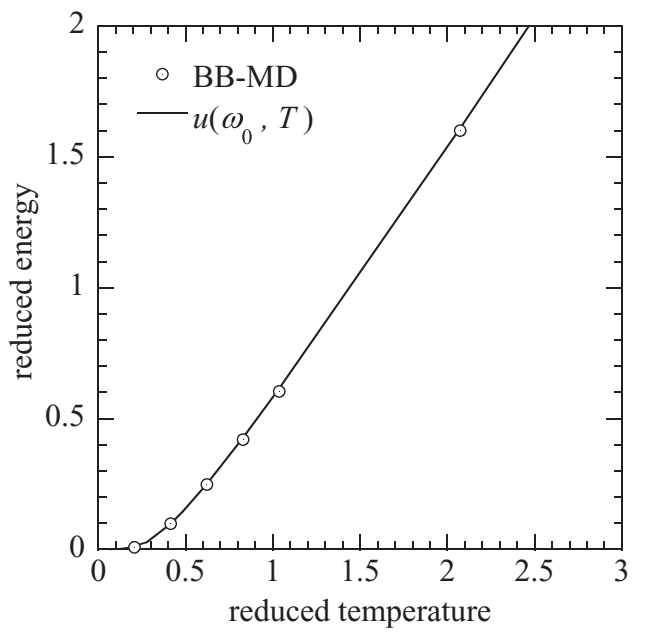

FIG. 2. Energy of a harmonic dipole. Comparison between the simulated energy (BB-MD) and the energy given by $u\left(\omega_{0}, T\right)$ as a function of temperature. Energy and temperature are normalized by $\hbar \omega_{0}$ and $\hbar \omega_{0} / k_{B}$, respectively.

simulations,

$$
\begin{aligned}
\alpha(\omega)= & \frac{1}{3 \varepsilon_{0} k_{B} T V}[\langle\mathbf{p}(0) \mathbf{p}(0)\rangle \\
& \left.+i \omega \int_{0}^{\infty} \exp (i \omega t)\langle\mathbf{p}(0) \mathbf{p}(t)\rangle d t\right],
\end{aligned}
$$

where $V$ is the volume of the NP. The polarizability spectrum is obtained by considering the polarization autocorrelation, whereas $g(\omega)$ is computed from the particle's velocity as

$$
g(\omega)=\lim _{N_{s} \rightarrow \infty} \frac{1}{k_{B} T N_{s} \delta t} \sum_{i} m_{i}\left|\mathbf{v}_{i}(\omega)\right|^{2},
$$

where $N_{s}$ is the number of time steps $\delta t$. At thermal equilibrium, the total vibrational energy can be estimated by performing an integration over the full density of states:

$$
U_{v}(T)=\int_{0}^{\infty} g(\omega) u(\omega, T) d \omega,
$$

whereas the contribution, $U_{p}(T)$, is obtained by solely considering the polar modes $\left(\omega_{p}\right)$ from Eq. (15),

$$
U_{p}(T)=3 \sum_{p} u\left(\omega_{p}, T\right)
$$

The factor 3 stands for the polar-modes degeneracies. Figures 3(a) and 3(b) report the polarizability spectra cal-

TABLE I. Irreductible decomposition of vibrational modes of $(\mathrm{MgO})_{n}$ nanoparticles according to the $T_{d}$ symmetry group. The degeneracy of $A_{1}, A_{2}, E, T_{1}$, and $T_{2}$ modes are 1, 1, 2, 3, and 3, respectively.

\begin{tabular}{lcrr}
\hline \hline$n$ & Irr. representation & $\begin{array}{c}\text { Vib. } \\
\text { modes }\end{array}$ & $\begin{array}{r}\text { Polar } \\
\text { modes }\end{array}$ \\
\hline 4 & $2 A_{1}+2 E+T_{1}+3 T_{2}$ & 18 & 9 \\
32 & $12 A_{1}+4 A_{2}+16 E+19 T_{1}+27 T_{2}$ & 186 & 81 \\
108 & $36 A_{1}+18 A_{2}+54 E+71 T_{1}+89 T_{2}$ & 642 & 267 \\
\hline \hline
\end{tabular}
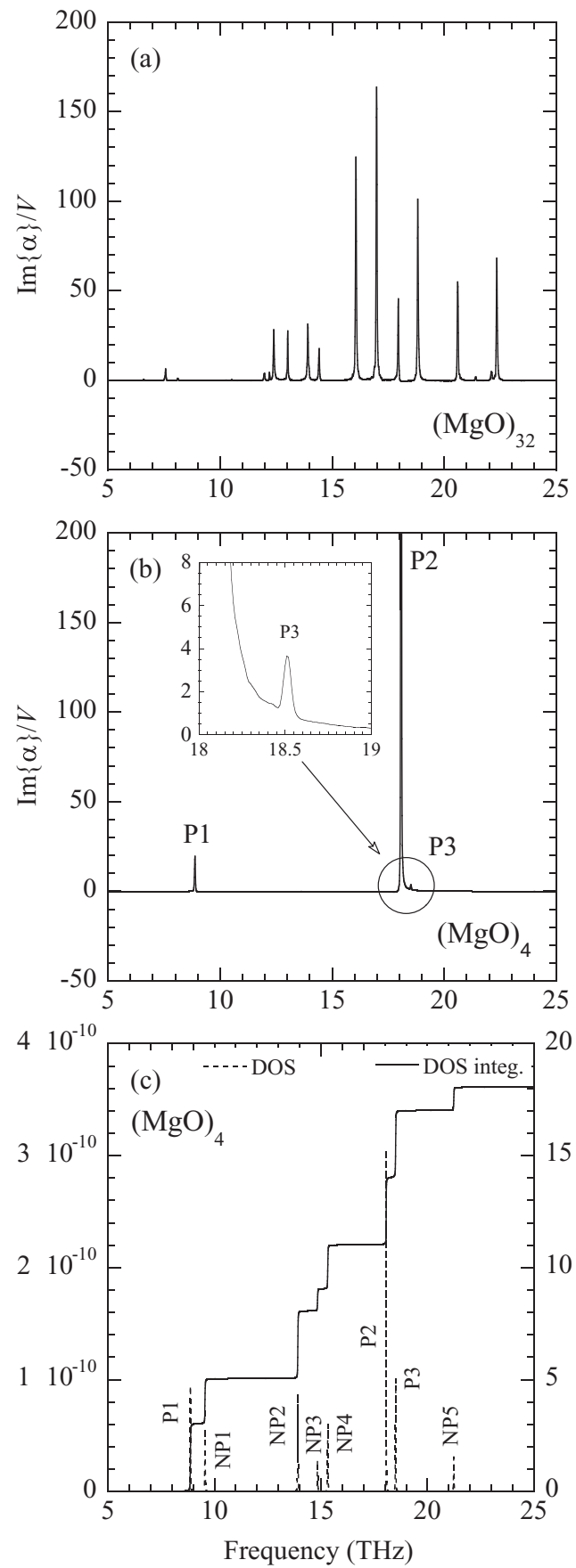

FIG. 3. (a), (b) $\operatorname{Im}\{\alpha(\omega)\}$ curves for $(\mathrm{MgO})_{32}$ and $(\mathrm{MgO})_{4}$ nanoparticles; (c) density of vibrational states (DOS) $g(\omega)$ and its integral for $(\mathrm{MgO})_{4}$. Polar $(\mathrm{P})$ and nonpolar $(\mathrm{NP})$ modes are labeled.

culated for $(\mathrm{MgO})_{4}$ and $(\mathrm{MgO})_{32}$ with 3 and 27 distinct polar frequencies, respectively. For $(\mathrm{MgO})_{4}$, the frequency $\mathrm{P} 2$ corresponds to the polariton resonance, that is the frequency at which IR absorption occurs the most efficiently. ${ }^{21}$

Figure 4 reports the temperature dependence of the vibrational energy directly obtained by MD simulations. The kinetic rotational energy of the NP is subtracted to its total energy for further comparison with Eq. (17). Results obtained for $(\mathrm{MgO})_{4},(\mathrm{MgO})_{32}$, and $(\mathrm{MgO})_{108}$ exhibited two trends: at low temperatures $(T<300 \mathrm{~K})$, BB-MD simulations fit nicely with 

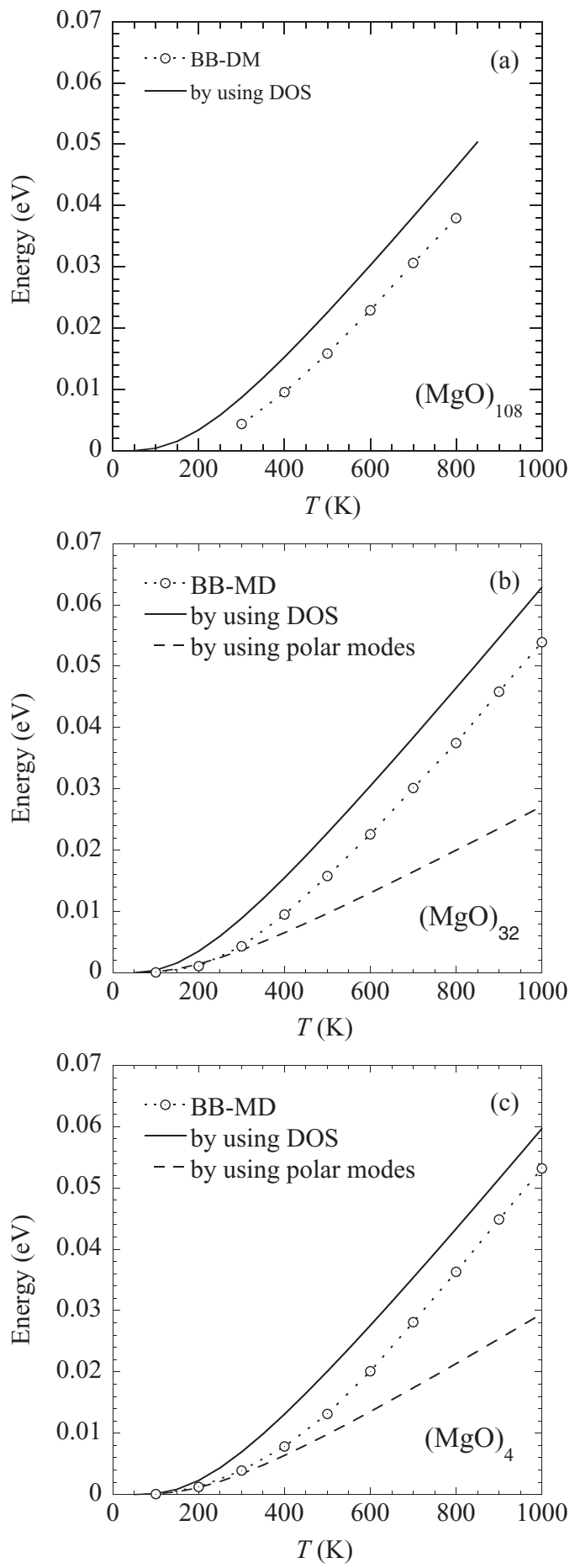

FIG. 4. Energy of $(\mathrm{MgO})_{n}$ nanoparticles at equilibrium as a function of temperature compared with energies $U_{v}(T)$ (solid line) and $U_{p}(T)$ (dashed line). The energy is normalized by the number of vibrational modes, $6 n-6$.

$U_{p}(T)$, whereas at higher temperatures, the predicted energy becomes larger than $U_{p}(T)$ but remains always lower than $U_{v}(T)$, which is unexpected.

Figures 4(b) and 4(c) show that the particle size extension enhances the contribution of nonpolar vibrational modes to the total energy, below $500 \mathrm{~K}$. These results prove that higher activation temperatures are required for a smaller particle to
TABLE II. Degeneracy, $g_{v}$, and symmetry of vibrational modes for the $(\mathrm{MgO})_{4}$ nanoparticle according to the $T_{d}$ symmetry group (Table I).

\begin{tabular}{lcccccccc}
\hline \hline & P1 & NP1 & NP2 & NP3 & NP4 & P2 & P3 & NP5 \\
\hline$g_{v}$ & 3 & 2 & 3 & 1 & 2 & 3 & 3 & 1 \\
Symm. & $T_{2}$ & $E$ & $T_{1}$ & $A_{1}$ & $E$ & $T_{2}$ & $T_{2}$ & $A_{1}$ \\
\hline \hline
\end{tabular}

allow an efficient vibrational relaxation of the absorbed energy. In other terms, inelastic scattering driven by anharmonicity of interatomic potential is too weak to redistribute the energy to the complete set of vibrational eigenmodes: this is a direct consequence of the discrete structure of the vibrational spectrum in a nanoscale cluster.

To get a better insight into the vibrational relaxation mechanisms occurring in isolated NPs, we consider the simple case of a $(\mathrm{MgO})_{4}$ for which only eight vibrational eigenfrequencies can be conveniently distinguished [Fig. 3(c)]. The three polar frequencies are indexed P1, P2, and P3 and nonpolar modes are named NP1 to NP5. The integration of $g(\omega)$ provides the degeneracies, $g_{v}$, of each frequency and by extension the number of vibrational degrees of freedom (Table II).

We propose to analyze the contribution of each vibrational mode to the total energy: at various temperatures, this contribution is evaluated with the integral

$$
\frac{1}{g_{v} N_{s} \delta t} \int_{\omega_{\min }}^{\omega_{\max }} \sum_{i} \frac{m_{i}\left|\mathbf{v}_{i}(\omega)\right|^{2}}{u(\omega, T)} d \omega
$$

where $\omega_{\max }$ and $\omega_{\min }$ are the bounds of the integration domain of each frequency mode. $u(\omega, T)$ is a normalization factor introduced to set the contribution of each mode between 0 and 1. This allows us to estimate how far this individual contribution is from its expected value. Numerical simulations have been performed during $10^{-8} \mathrm{~s}$ with a coupling coefficient $Z$ set at $10^{4}$. The steady-state regime was obtained within time ranges shorter than $200 \mathrm{ps}$.

Figure 5 reports the contribution rates obtained with a BB temperature ranging between 200 and $1000 \mathrm{~K}$. At $300 \mathrm{~K}$, it can be seen that the thermal energy is mostly carried by

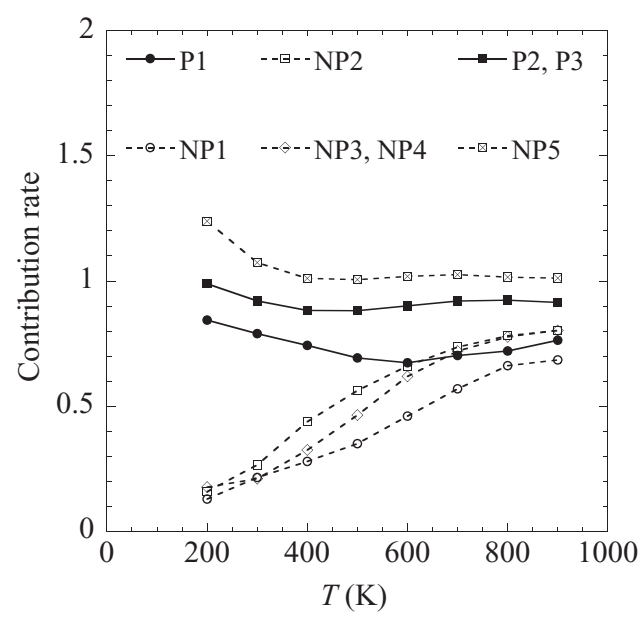

FIG. 5. Contribution rate of each vibrational mode to the energy of the $(\mathrm{MgO})_{4}$ nanoparticle at various temperatures. 
the polar modes P1, P2, and P3 and NP5. NP5 corresponds to the breathing mode and its activation is attributed to the effect of thermal expansion of the nanoparticle. P1 tends to decrease when the $\mathrm{BB}$ temperature increases, indicating that a part of its vibrational energy has been transmitted to the modes identified. Except for NP5 (21,2 THz), these contributions remain almost negligible below $400 \mathrm{~K}$ : they do not contribute to the total energy. In what follows, we analyze the mechanisms responsible for inelastic modal coupling allowing the vibrational relaxation in this system and also the reasons that prevent an efficient energy redistribution among all modes.

\section{VIBRATIONAL SPECTROSCOPY AND RELAXATION PATHWAY FOR $(\mathrm{MgO})_{4}$}

This section discusses and demonstrates how the vibrational relaxation channels of a $(\mathrm{MgO})_{4}$ can be studied in detail. The idea is to look at the response of the crystal when performing selective monochromatic excitation with a deterministic electric field tuned on each polar eigenfrequency. The amount of energy absorbed optically is thus perfectly known as well as its fraction relaxed preferentially toward vibrational modes.

To assess the inelastic scattering contribution, we only need to measure this fraction as a function of the system temperature. We thus performed monochromatic absorption simulations at 300 and $600 \mathrm{~K}$ for the $(\mathrm{MgO})_{4}$ nanoparticle. At $300 \mathrm{~K}$, we found that there is only one relaxation channel from polar modes. As reported in Table III, this relaxation corresponds to the mixing

$$
\hbar \omega_{\mathrm{P} 3} \simeq 2 \hbar \omega_{\mathrm{NP} 1}
$$

which is dominant. As discussed earlier, P2 corresponds to the polariton resonance and consequently absorption is dominant at this frequency [see Fig. 3(b)]. A large fraction of the thermal energy absorbed from the BB cavity remains trapped in this mode, since no mixing rule involving this frequency has been identified at this temperature.

At higher temperature $(600 \mathrm{~K})$, the relaxation given by the rule [Eq. (20)] remains the most important; however, complementary processes are involved. NP3 and NP5 are obtained according to

$$
2 \hbar \omega_{\mathrm{P}_{2}} \simeq \hbar \omega_{\mathrm{NP}_{3}}+\hbar \omega_{\mathrm{NP}_{5}},
$$

TABLE III. Fraction of energy relaxed per mode (\%) when exciting at frequencies P1, P2, and P3 (Table II).

\begin{tabular}{ccccccccc}
\hline \hline & P1 & NP1 & NP2 & NP3 & NP4 & P2 & P3 & NP5 \\
\hline & & & \multicolumn{7}{c}{$300 \mathrm{~K}$} \\
P1 & 99.75 & 0 & 0 & 0 & 0 & 0.25 & 0 & 0 \\
P2 & 0 & 0 & 0 & 0 & 0 & 100 & 0 & 0 \\
P3 & 0.15 & 99.42 & 0 & 0 & 0 & 0 & 0.44 & 0 \\
& & & & $600 \mathrm{~K}$ & & & & \\
P1 & 99.4 & 0 & 0 & 0 & 0 & 0.5 & 0 & 0 \\
P2 & 1.0 & 3.4 & 0.2 & 0.3 & 0 & 92.4 & 2.2 & 0.4 \\
P3 & 2.3 & 91.8 & 0 & 0 & 0 & 0 & 5.9 & 0 \\
\hline \hline
\end{tabular}

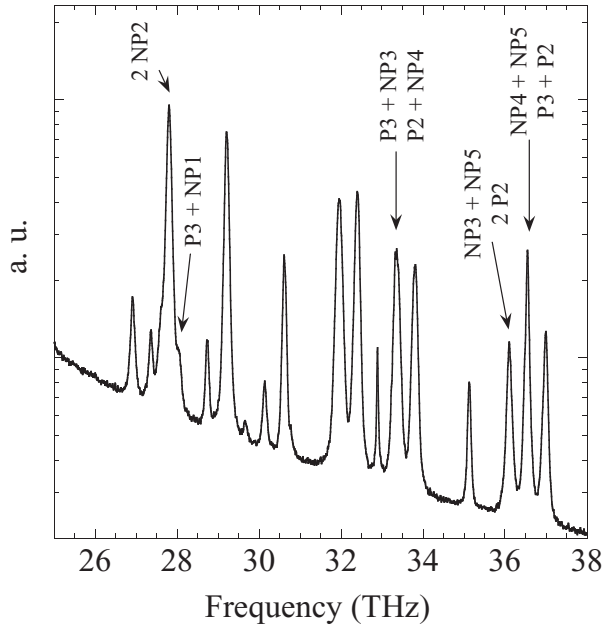

FIG. 6. Anharmonic vibrational spectrum obtained from Eq. (16) and corresponding to a magnification of the $g(\omega)$ at higher frequency regions.

which is consistent with the fact that, when exciting P2, the amount of energy relaxed to NP3 and NP5 is roughly the same. Moreover, when exciting at the frequency P3, one obtains

$$
\hbar \omega_{\mathrm{P}_{3}} \simeq 2 \hbar \omega_{\mathrm{NP}_{2}}-\hbar \omega_{\mathrm{NP}_{1}}
$$

The complete set of allowed inelastic processes is checked by considering the anharmonic contribution of the vibrational spectrum (Fig. 6). Additional contribution from NP4 and NP5 to the total vibrational has been identified with the following rules:

$$
\begin{aligned}
\hbar \omega_{\mathrm{P}_{2}}+\hbar \omega_{\mathrm{NP}_{4}} & \simeq \hbar \omega_{\mathrm{P}_{3}}+\hbar \omega_{\mathrm{NP}_{3}}, \\
\hbar \omega_{\mathrm{P}_{3}}+\hbar \omega_{\mathrm{P}_{2}} & \simeq \hbar \omega_{\mathrm{NP}_{4}}+\hbar \omega_{\mathrm{NP}_{5}} .
\end{aligned}
$$

In summary, by performing selective mode excitation and by identifying the responding modes, we were able to reconstruct the vibrational relaxation channels of a $(\mathrm{MgO})_{4}$. We found that the relaxation of the thermal energy absorbed at the frequency P3 is the most efficient. On the other hand, $\mathrm{P} 2$, which dominates the radiation absorption, do not transfer any energy to the rest of the system. This energy trapping effect explains the behavior observed on Fig. 4, where the total energy of the NP remained always lower than the expected equilibrium value. This result is clearly a consequence of size confinement. Indeed, the discrete structure of the vibrational spectrum introduces selection rules for relaxation. As bulk materials exhibit band structure, this effect can be observed only in nanostructures.

\section{CONCLUSION}

We have introduced a model to study the radiative heat transfer by molecular-dynamics simulations using a BB as a thermostat for dielectric nanoparticles. This model allows the recovery of the low-temperatures vibrational dynamics for $(\mathrm{MgO})_{n}$ nanoparticles. By investigating their heating 
mechanisms with the $\mathrm{BB}$, we have found situations where the vibrational relaxation in size-confined dielectrics strongly differs from bulk materials. Thermal energy absorption is clearly mediated by polar modes, and absorption is predominant at the polariton resonance. We observed the trapping of heat at this particular frequency and identified this effect as a consequence of size confinement. Our results clearly unveil important differences between the true low-temperature behavior of nanomaterials, and the approximations typically used above Debye temperature.

\section{ACKNOWLEDGMENTS}

H.D. gratefully acknowledges helpful discussions with G. Baldinozzi and G. Geneste about symmetry and vibrational properties of nanoparticles.

\section{APPENDIX: GENERATION OF THE RANDOM FIELD}

This part presents the technique used to generate the fluctuating electric field $E(t)$ in the time domain assuming a Planck-law power density spectrum. ${ }^{18}$ Since it models the thermal radiation emitted by a BB cavity, $E(t)$ is a random Gaussian variable. The first- and second-order moments can be written as

$$
\begin{gathered}
\langle E(t)\rangle=0, \\
\langle E(t) E(t+\tau)\rangle=\int_{-\infty}^{+\infty} I_{E}(|\omega|, T) e^{-i \omega \tau} \frac{d \omega}{2 \pi},
\end{gathered}
$$

where $I_{E}$ is given by Eq. (4).

The description of the fluctuating field in the time domain is expressed in a discrete form, $t_{n}=n \delta t$, where $n=1, \ldots, N$. We write the electric field as a sum of independent random Gaussian variables $(X)$ as

$$
E_{n}=E(n \delta t)=\sum_{j=-\infty}^{+\infty} W_{j} X_{j+n},
$$

with $X$ having the following properties:

$$
\begin{aligned}
\left\langle X_{i}\right\rangle & =0, \\
\left\langle X_{i} X_{j}\right\rangle & =\delta_{i j} .
\end{aligned}
$$

Now, $W_{j}$ are the unknown variables to build the expected correlation function. We have

$$
\begin{aligned}
\left\langle E_{i}\right\rangle & =0, \\
\left\langle E_{i} E_{j}\right\rangle & =\delta_{i j},
\end{aligned}
$$

*hichem.dammak@ecp.fr

${ }^{1}$ V. P. Carey, G. Chen, C. Grigoropoulos, M. Kaviany, and A. Majumdar, Nanoscale Microscale Thermophys. Eng. 12, 1 (2008).

${ }^{2}$ K. Schwab, E. A. Henriksen, J. M. Worlockaznd, and M. L. Roukes, Nature (London) 404, 974 (2000).

${ }^{3}$ V. Myroshnychenko, J. Rodriguez-Fernandez, and I. PastorizaSantos, Chem. Soc. Rev. 37, 1792 (2008). so that we are looking for a series having the following property:

$$
\sum_{j=-\infty}^{\infty} W_{j} W_{j-l}=\int_{-\infty}^{\infty} I_{E}(\omega, T) e^{-i \omega t_{l}} \frac{d \omega}{2 \pi} .
$$

$W_{j}$ is even and real and the Fourier transform of $W(t)$ obeys the equation $\tilde{W}(\omega)=\tilde{W}(-\omega)$. As a result, we obtain

$$
\tilde{W}(\omega)=\sqrt{\delta t} \sqrt{I_{E}(\omega)} .
$$

The transformation of the $W_{j}$ in the time domain gives

$$
W\left(t_{j}\right)=\sqrt{\delta t} \int_{-\infty}^{+\infty} \sqrt{I_{E}(\omega, T)} e^{-i \omega t_{j}} \frac{d \omega}{2 \pi} .
$$

For practical reasons, $E\left(t_{n}\right)$ is written according to a discrete Fourier transform (DFT). The DFT of $W_{j}$ and $X_{j}$ are first introduced as

$$
\begin{aligned}
& W_{j}=\frac{1}{\sqrt{N}} \sum_{l=-N / 2+1}^{N / 2} \tilde{W}_{l} e^{-\frac{i 2 \pi j l}{N}}, \\
& X_{j}=\frac{1}{\sqrt{N}} \sum_{l=-N / 2+1}^{N / 2} \tilde{X}_{l} e^{-\frac{i 2 \pi j l}{N}},
\end{aligned}
$$

then $E$ takes the form

$$
E\left(t_{j}\right)=\sum_{l=-N / 2+1}^{N / 2} \tilde{W}_{-l} \tilde{X}_{l} e^{-\frac{i 2 \pi j l}{N}} .
$$

Taking the integral form of Eq. (A9),

$$
W_{j}=\frac{1}{\sqrt{N} \delta \omega} \int_{-\infty}^{+\infty} \tilde{W}(\omega) e^{-i t_{j} \omega} d \omega,
$$

where $N \delta \omega \delta t=2 \pi$. This latter equation can be identified with Eq. (A8) and one obtains

$$
\tilde{W}_{l}=\frac{1}{\sqrt{N \delta t}} \sqrt{I_{E}\left(\omega_{l}\right)},
$$

where $\omega_{l}=l \delta \omega . E\left(t_{j}\right)$ can be finally written as

$$
E\left(t_{j}\right)=\frac{1}{\sqrt{N \delta t}} \sum_{l=-N / 2+1}^{N / 2} \sqrt{I_{E}\left(\omega_{l}\right)} \tilde{X}_{l} e^{-\frac{i 2 \pi j l}{N}},
$$

where

$$
\tilde{X}_{l}=\frac{1}{\sqrt{2}}\left(\tilde{M}_{l}+i \tilde{N}_{l}\right) .
$$

Note that $\tilde{M}_{l}$ and $\tilde{N}_{l}$ are also independent Gaussian variables and $\tilde{M}_{-l}=\tilde{M}_{l}, \tilde{N}_{-l}=-\tilde{N}_{l}$ in order to obtain real values for $X_{j}$.
${ }^{4}$ D. M. Grimes and C. A. Grimes, J. Nanosci. Nanotechnol. 2, 545 (2002).

${ }^{5}$ G. Domingues, S. Volz, K. Joulain, and J. J. Greffet, Phys. Rev. Lett. 94, 085901 (2005).

${ }^{6}$ R. Carminati and J. J. Greffet, Phys. Rev. Lett. 82, 1660 (1999).

${ }^{7}$ A. V. Shchegrov, K. Joulain, R. Carminati, and J. J. Greffet, Phys. Rev. Lett. 85, 1548 (2000).

${ }^{8}$ S. A. Biehs and J. J. Greffet, Phys. Rev. B 81, 245414 (2010). 
${ }^{9}$ Y. Gao, A. Marconnet, M. Panzer, S. LeBlanc, S. Dogbe, Y. Ezzahri, A. Shakouri, and K. Goodson, J. Electron. Mater. 39, 1456 (2010). ${ }^{10}$ M. Laroche, R. Carminati, and J. J. Greffet, J. Appl. Phys. 100, 63704 (2006).

${ }^{11}$ M. J. Uline, D. W. Siderus, and C. David, J. Chem. Phys. 128, 126301 (2008).

${ }^{12}$ S. Merabia, P. Keblinski, L. Joly, L. J. Lewis, and J. L. Barrat, Phys. Rev. E 79, 021404 (2009).

${ }^{13}$ D. Lacroix, K. Joulain, and D. Lemonnier, Phys. Rev. B 72, 064305 (2005).

${ }^{14}$ A. J. H. McGaughey and M. Kaviany, Phys. Rev. B 69, 094303 (2004).
${ }^{15}$ H. Zhong and J. R. Lukes, Phys. Rev. B 74, 125403 (2006).

${ }^{16}$ Y. F. Chen, D. Y. Li, J. R. Lukes, Z. H. Ni, and M. H. Chen, Phys. Rev. B 72, 174302 (2005).

${ }^{17}$ H. M. Miller, Proc. Natl. Acad. Sci. USA 102, 6660 (2005).

${ }^{18}$ A. A. Maradudin, T. Michel, A. R. McGurn, and E. R. Mndez, Ann. Phys. (Leipzig) 203, 255 (1990).

${ }^{19}$ J. D. Jackson, Classical Electrodynamics, 3rd ed. (John Wiley \& Sons, New York, 1999).

${ }^{20}$ M. Matsui, J. Chem. Phys. 91, 489 (1989).

${ }^{21}$ C. F. Bohren and D. R. Huffman, Absorption and Scattering of Light by Small Particles (John Wiley \& Sons, New York, 1983). 